\title{
In vitro effects of compounds isolated from Sideritis brevibracteata on bovine kidney cortex glutathione reductase
}

\author{
Berivan Tandogan¹, Ayşegül Güvenç², İhsan Çalış3 and Nuriye Nuray Ulusu ${ }^{1 凶}$ \\ 'Department of Biochemistry, Faculty of Medicine, Hacettepe University, Ankara, Turkey; 2Department of Pharmaceutical Botany, Faculty of \\ Pharmacy, Ankara University, Ankara, Turkey; ${ }^{3}$ Department of Pharmacognosy, Faculty of Pharmacy, Hacettepe University, Ankara, Turkey
}

\begin{abstract}
Glutathione reductase (GR, E.C 1.6.4.2) is a flavoprotein that catalyzes NADPH-dependent reduction of oxidized glutathione (GSSG) to reduced glutathione (GSH). The aim of this study was to investigate in vitro effects of phenolic compounds isolated from Sideritis brevibracteata on bovine kidney GR. The Sideritis species are widely found in nature and commonly used as medicinal plants. 7-O-glycosides of 8-OH-flavones (hypolaetin, isoscutellarein and 3 '-hydroxy-4'-O-methylisoscutellarein) were isolated from aerial parts of Sideritis brevibracteata. These compounds inhibited bovine kidney cortex GR in a concentration-dependent manner. Kinetic characterization of the inhibition was also performed.
\end{abstract}

Keywords: glutathione reductase; inhibition; kinetic; Sideritis brevibracteata; 8-hydroxyflavone glycosides

Received: 19 April, 2010; revised: 10 December, 2010; accepted: 28 November, 2011; available on-line: 06 December, 2011

\section{INTRODUCTION}

The research on natural compounds from medicinal plants and their synthetic analogues has accelerated in recent years due to their importance in drug discovery using a medicinal chemistry approach (Lee, 2010). In this study, we investigated in vitro effects of the compounds isolated from Sideritis brevibracteata P. H. Davis on glutathione reductase (GR). Sideritis L. (Lamiaceae=Labiatae) species are widely found in nature and are commonly used as medicinal plants and herbal teas in the Mediterranean region (Güvenç et al., 2005). Sideritis species contain mainly flavonoids, essential oils, diterpenes, phenylpropanoid glycosides, and iridoid glucosides (Ezer et al., 1992; Baser et al., 1997). Antibacterial, anti-inflammatory, antinociceptive, anti-oxidant, and aldose reductase inhibitory activities of compounds from this genus have been reported (Barberan et al., 1987; Güvenç et al., 2010). These biological activities are mainly attributed to the flavonoid content of this plant (Yesilada \& Ezer, 1989; Rios et al., 1992; De Las Heras et al., 1994).

Glutathione reductase (GR) reduces glutathione disulfide (GSSG) to its thiol form GSH (Arscott et al., 2000). Alterations in the activity of GR may affect the cellular defense system and cause oxidative stress in the cell. Inhibition of GR decreases the GSH/GSSG ratio, and this contributes to oxidative stress and the pathogenesis of many diseases. GR is a homodimeric flavoenzyme, and cysteine, histidine and tyrosine are amino acids critical for the GR activity. The redox properties of this enzyme can be inhibited or activated by several chemicals, drugs or natural substrates (Untucht-Grau et al., 1981; Bauer et al., 2006; Krauth-Siegel et al., 1998).

Glutathione reductase (GR) is an important housekeeping enzyme for redox homeostasis both in human cells and in the causative agent of tropical malaria. Its inhibitors are usually considered as drug sensitizers: they do not display high intrinsic antimalarial or antitumoral activities when used alone, but they can enhance the effects of chloroquine or cytotoxic agents (Bauer et al., 2006). Acetylated allose containing 8-hydroxyflavone glycosides and a phenylethanoid glycoside have been isolated from aerial parts of S. brevibracteata (Güvenç et al., 2010). The present study aimed to investigate the in vitro effects of the phenolic compounds on bovine kidney cortex GR.

\section{MATERIALS AND METHODS}

Materials. Nicotinamide adenine dinucleotide phosphate reduced form (NADPH) and oxidized glutathione (GSSG) were obtained from Sigma Chemical Co., MO, USA.

Plant material. Aerial parts of S. brevibracteata P.H. Davis were collected from C3 Antalya: Alanya, AlanyaTürbelinaz road, around Bektas, calcareous rocks, in macchie, $200 \mathrm{~m}, \mathrm{~N}$ 36_ 33.5600, E 32_ 00 4840, by Hayri Duman, 27.05.2001. Voucher specimens (coll. Hayri Duman 875) are deposited in the Botany Department Herbarium at Gazi University (GAZI), Turkey.

Extraction and isolation. The air-dried and powdered aerial parts of $S$. brevibracteata $(350 \mathrm{~g})$ were extracted with $3500 \mathrm{ml} \mathrm{MeOH}$ in a Soxhlet apparatus for $12 \mathrm{~h}$. The $\mathrm{MeOH}$ extract was then evaporated in vacuum $\left(40^{\circ} \mathrm{C}\right)$. The residue $(82.80 \mathrm{~g})$ was dissolved in $500 \mathrm{ml} \mathrm{H}$ O-MeOH mixture $(9: 1, \mathrm{v} / \mathrm{v})$, then partitioned three times with chloroform $(3 \times 250 \mathrm{ml})$, AcOEt $(3 \times 250 \mathrm{ml})$ and $n$-BuOH saturated with water $(3 \times 250 \mathrm{ml})$. These three phases were evaporated under reduced pressure and total extracts of chloroform $(10.50 \mathrm{~g})$, AcOEt $(9.38 \mathrm{~g})$ and $n$-BuOH (25.11 g) were obtained. An aliquot of $n-\mathrm{BuOH}$ extract $(2.72 \mathrm{~g}$ ) was subjected to medium-pressure liquid chromatography (MPLC), using water $(200 \mathrm{ml})$ and $\mathrm{H}_{2} \mathrm{O}-\mathrm{MeOH}$ mixtures $(5 \rightarrow 45 \%$ $\mathrm{MeOH}$ in steps of $5 \%$ of $\mathrm{MeOH}$ (each $200 \mathrm{ml})$ ). Flavonoids were eluted in the following order: [1] (88 mg) (fractions 61-62), [2] (142 mg) (fractions 65-69), [4] (292 mg) (fractions 79-81), [5] (118 mg) (fractions 82-83) and [6] (44 mg) (fractions 86-87). Their structures were identified as hypolaetin $7-O-[6$ "'-O-acetyl- $\beta$-D-allopyranosyl- $(1 \rightarrow 2)]-\beta$ -

\footnotetext{
e-mail: nnulusu@hacettepe.edu.tr
}

Abbreviations: GR, glutathione reductase; GSH, reduced glutathione; GSSG, oxidized glutathione; NADPH, nicotinamide adenine dinucleotide phosphate. 
D-glucopyranoside [1], isoscutellarein 7-O-[6"'-O-acetyl- $\beta$-Dallopyranosyl- $(1 \rightarrow 2)]-\beta$-D-glucopyranoside [2], hypolaetin 7-O-[6"'-O-acetyl- $\beta$-D-allopyranosyl- $(1 \rightarrow 2)]-6$ "-O-acetyl- $\beta$-Dglucopyranoside [4], isoscutellarein 7-O-[6"'-O-acetyl- $\beta$-Dallopyranosyl- $(1 \rightarrow 2)]-6$ "- $O$-acetyl- $\beta$-D-glucopyranoside [5], and 3'-hydroxy-4'-O-methylisoscutellarein 7-O-[6"'-O-acetyl$\beta$-D-allopyranosyl- $(1 \rightarrow 2)]-6$ "- $O$-acetyl- $\beta$-D-glucopyranoside [6] (Güvenç et al., 2010).

Assay of glutathione reductase. Glutathione reductase (GR) activity was determined according to the modified Stall method (Acan \& Tezcan, 1989). The incubation mixture contained $100 \mathrm{mM}$ sodium phosphate buffer, $\mathrm{pH}$ 7.4; $1 \mathrm{mM}$ GSSG; $0.2 \mathrm{mM}$ NADPH; and bovine kidney cortex GR. Decrease in the absorbance of NADPH at $340 \mathrm{~nm}$ was monitored spectrophotometrically at $37^{\circ} \mathrm{C}$.

A unit of activity (U) was defined as the amount of enzyme that catalyzes the oxidation of $1 \mu$ mole of NADPH in 1 min under these conditions.

Purification of bovine glutathione reductase. Bovine kidney cortex GR was purified by two subsequent chromatography steps after ultracentrifugation and heat denaturation: 2',5'-ADP Sepharose 4B affinity and DEAE Sepharose Fast Flow anion exchange chromatography. All the procedures were carried out at $+4^{\circ} \mathrm{C}$. GR was purified $34806-$ fold with a final yield of $85 \%$ and specific activity of 727 $\mathrm{U} / \mathrm{mg}$ using this method (Tandogan \& Ulusu, 2010).

Inhibition studies. Activities were measured by addition of the compounds investigated at different concentrations to the assay mixture given above for GR measurement. GR assays in the presence of the compounds were performed without enzyme-inhibitor preincubation, and the reactions were initiated by adding the enzyme to a substrate-inhibitor mixture.

Statistical analysis of kinetic data. The data were analyzed and the kinetic constants were calculated using the equations described in (Segel, 1975) by means of a nonlinear curve-fitting program of Statistica.

\section{RESULTS}

\section{Inhibition of bovine kidney cortex GR by hypolaetin 7-O-[6"'-O-acetyl- $\beta$-D-allopyranosyl- $(1 \rightarrow 2)]-\beta$-D- glucopyranoside [1]}

This compound inhibited bovine kidney cortex GR in a concentration-dependent manner with an inhibi-

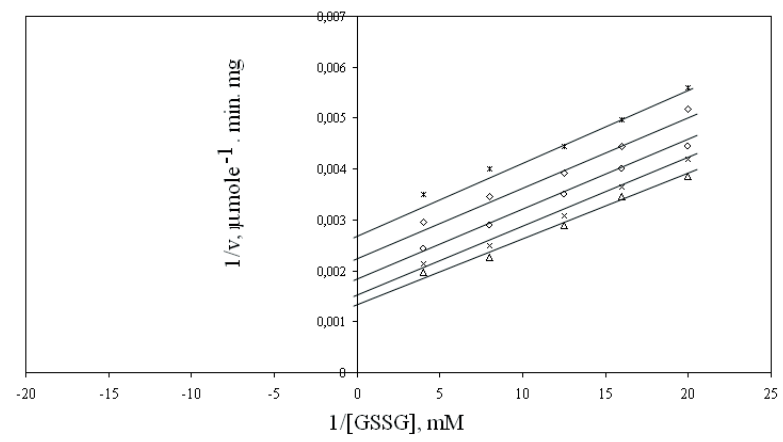

Figure 1. Inhibition of bovine kidney cortex glutathione reductase by hypolaetin 7-O-[6"'-O-acetyl- $\beta$-D-allopyranosyl- $(1 \rightarrow 2)]-\beta$ D-glucopyranoside.

Lineweaver-Burk double reciprocal plot of initial velocity against GSSG as varied substrate and compound [1] as inhibitor at a fixed $\mathrm{NADPH}(0.1 \mathrm{mM})$ concentrations. $(\mathrm{x}) 0.1 \mathrm{mM} ;(\diamond) 0.15 \mathrm{mM}$; (o) 0.2 $\mathrm{mM} ;\left(^{*}\right) 0.25 \mathrm{mM}$ compound [1].

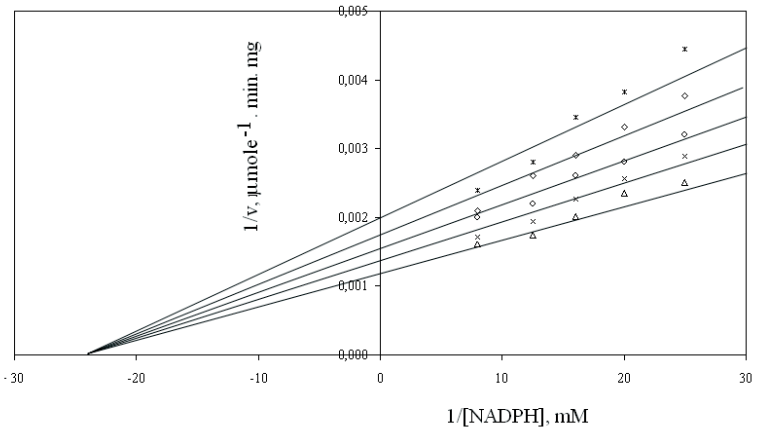

Figure 2. Inhibition of bovine kidney cortex glutathione reductase by hypolaetin 7-O-[6"'-O-acetyl- $\beta$-D-allopyranosyl-( $1 \rightarrow 2)]$ $\beta$-D- glucopyranoside.

Lineweaver-Burk double reciprocal plot of initial velocity against NADPH as varied substrate and compound [1] as inhibitor at a fixed GSSG (0.7 mM) concentrations. (x) $0.1 \mathrm{mM}$; ( () $0.15 \mathrm{mM}$; (o) $0.2 \mathrm{mM} ;\left(^{*}\right) 0.25 \mathrm{mM}$ compound [1].

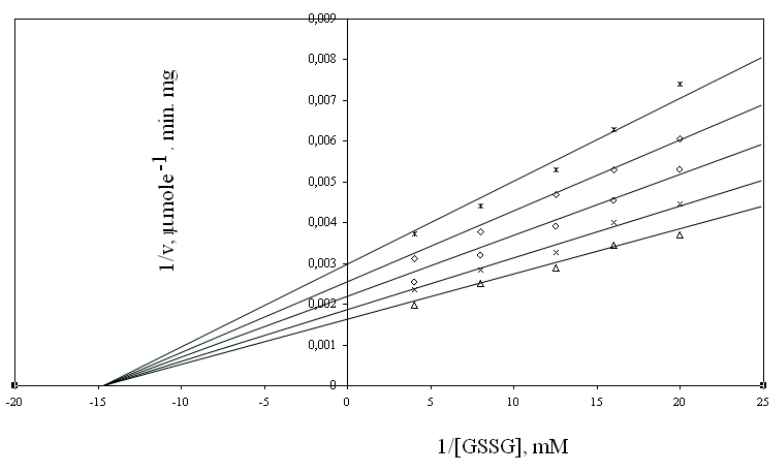

Figure 3. Inhibition of bovine kidney cortex glutathione reductase by isoscutellarein 7-O-[6"'-O-acetyl- $\beta$-D-allopyranosyl$(1 \rightarrow 2)]-\beta-D$ - glucopyranoside.

Lineweaver-Burk double reciprocal plot of initial velocity against GSSG as varied substrate and compound [2] as inhibitor at a fixed NADPH $(0.1 \mathrm{mM})$ concentrations. $(\mathrm{x}) 0.1 \mathrm{mM} ;(\diamond) 0.2 \mathrm{mM}$; $(\mathrm{o}) 0.25$ $\mathrm{mM} ;\left(^{*}\right) 0.3 \mathrm{mM}$ compound [2]

tory concentration $\left(\mathrm{IC}_{50}\right)$ value of $0.177 \pm 0.02 \mathrm{mM}$. The effects of compound [1] $(0.1 ; 0.15 ; 0.2 ; 0.25 \mathrm{mM})$ on GR from bovine kidney cortex were investigated using Lineweaver-Burk double reciprocal plot, and initial velocity data were analyzed by a nonlinear curve fitting program. The mode of inhibition of GR by compound [1] was determined as uncompetitive with respect

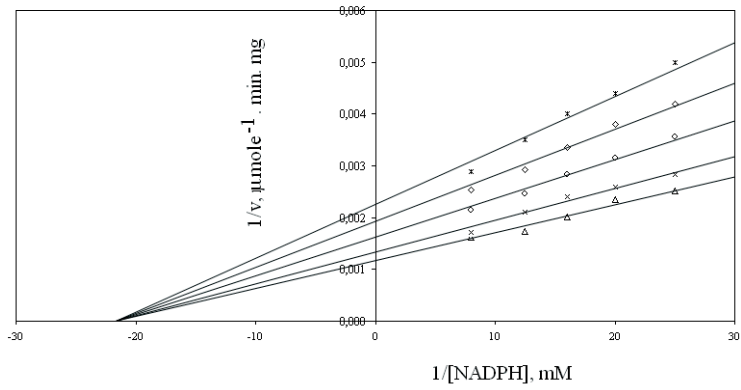

Figure 4. Inhibition of bovine kidney cortex glutathione reductase by isoscutellarein 7-O-[6"'-O-acetyl- $\beta$-D-allopyranosyl$(1 \rightarrow 2)]-\beta$-D-glucopyranoside.

Lineweaver-Burk double reciprocal plot of initial velocity against NADPH as varied substrate and compound [2] as inhibitor at a fixed GSSG (0.7 mM) concentrations. (x) $0.1 \mathrm{mM} ;(\diamond) 0.2 \mathrm{mM}$; (o) $0.25 \mathrm{mM} ;\left(^{*}\right) 0.3 \mathrm{mM}$. 


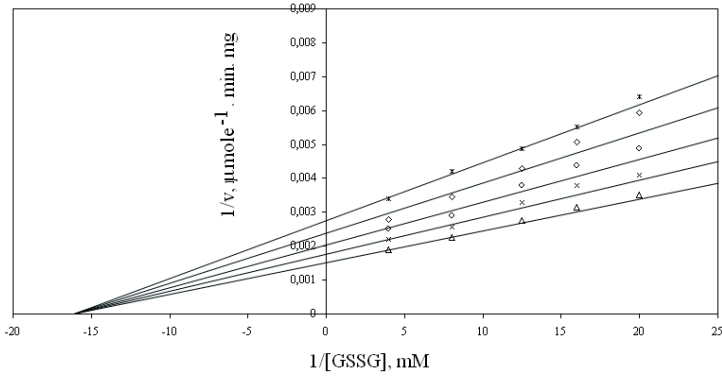

Figure 5. Inhibition of bovine kidney cortex glutathione reductase by hypolaetin 7-O-[6"'-O-acetyl- $\beta$-D-allopyranosyl- $(1 \rightarrow 2)]-\beta$ D-glucopyranoside.

Lineweaver-Burk double reciprocal plot of initial velocity against GSSG as varied substrate and compound [4] as inhibitor at a fixed NADPH (0.1 mM) concentrations. (x) $0.1 \mathrm{mM}$; ( ()$) 0.15 \mathrm{mM}$; (o) 0.2 $\left.\mathrm{mM} ;{ }^{*}\right) 0.25 \mathrm{mM}$ compound [4]

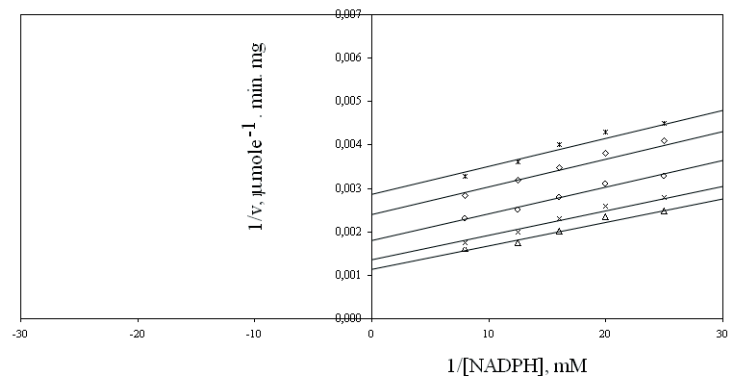

Figure 6. Inhibition of bovine kidney cortex glutathione reductase by hypolaetin 7-O-[6"'-O-acetyl- $\beta$-D-allopyranosyl-( $1 \rightarrow 2)]-\beta$ D-glucopyranoside.

Lineweaver-Burk double reciprocal plot of initial velocity against NADPH as varied substrate and compound [4] as inhibitor at a fixed GSSG (0.7 mM) concentrations. (x) $0.1 \mathrm{mM} ;(0) 0.15 \mathrm{mM}$; (o) $0.2 \mathrm{mM} ;\left(^{*}\right) 0.25 \mathrm{mM}$ compound [4].

to GSSG (Fig. 1) and noncompetitive with respect to NADPH (Fig. 2). Inhibition constants were calculated as $K_{i \mathrm{GSSG}} 0.453 \pm 0.05 \mathrm{mM}$ and $K_{\mathrm{NADPH}} 0.465 \pm 0.04 \mathrm{mM}$.

Inhibition of bovine kidney cortex GR by isoscutellarein 7-O-[6"'-O-acetyl- $\beta$-D-allopyranosyl- $(1 \rightarrow 2)]-\beta$-Dglucopyranoside [2]

Isoscutellarein $\quad 7-O-[6 " '-O$-acetyl- $\beta$ - $D$-allopyranosyl$(1 \rightarrow 2)]$ - $\beta$ - $D$-glucopyranoside inhibited $\mathrm{GR}$, with an $\mathrm{IC}_{50}$

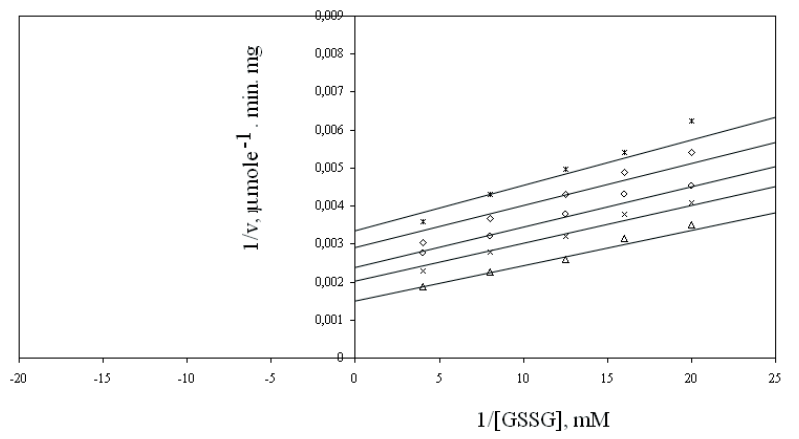

Figure 7. Inhibition of bovine kidney cortex glutathione reductase by isoscutellarein 7-O-[6"'-O-acetyl- $\beta$-D-allopyranosyl$(1 \rightarrow 2)]-\beta$-D-glucopyranoside.

Lineweaver-Burk double reciprocal plot of initial velocity against GSSG as varied substrate and compound [5] as inhibitor at a fixed NADPH (0.1 mM) concentrations. (x) 0.05; mM ( 0 ) $0.1 \mathrm{mM}$; (o) 0.2 $\left.\mathrm{mM} ;{ }^{*}\right) 0.3 \mathrm{mM}$ compound [5].

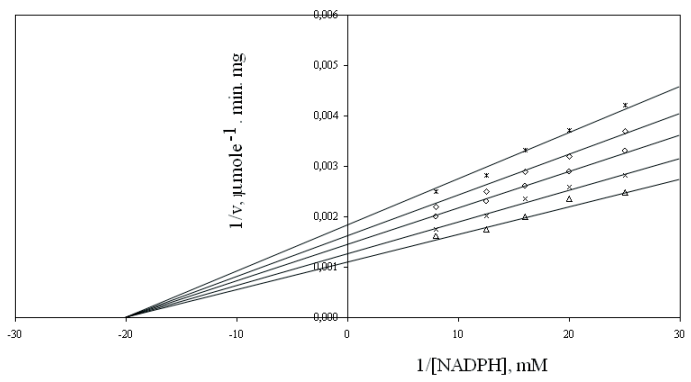

Figure 8. Inhibition of bovine kidney cortex glutathione reductase by isoscutellarein 7-O-[6"'-O-acetyl- $\beta$-D-allopyranosyl$(1 \rightarrow 2)]-\beta$-D-glucopyranoside.

Lineweaver-Burk double reciprocal plot of initial velocity against GSSG as varied substrate and compound [5] as inhibitor at a fixed GSSG (0.7 mM) concentrations. (x) $0.05 \mathrm{mM}$; ( 0$) 0.1 \mathrm{mM}$; (o) 0.2 $\mathrm{mM} ;\left(^{*}\right) 0.3 \mathrm{mM}$ compound [5].

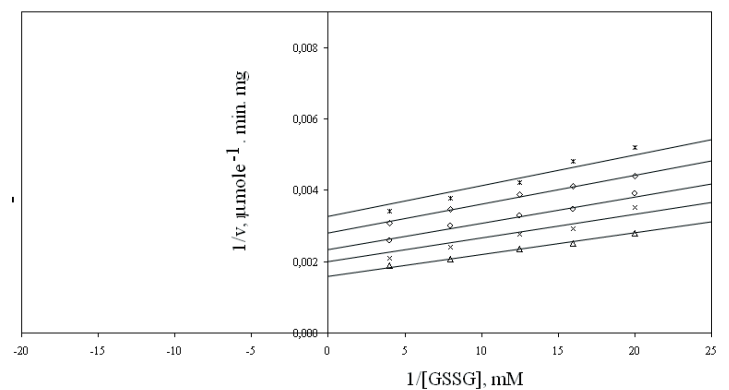

Figure 9. Inhibition of bovine kidney cortex glutathione reductase by $3^{\prime}$-hydroxy-4'-O-methylisoscutellarein 7-O-[6"'-O-acetyl- $\beta$ D-allopyranosyl- $(1 \rightarrow 2)]-\beta$-D-glucopyranoside.

Lineweaver-Burk double reciprocal plot of initial velocity against GSSG as varied substrate and compound [6] as inhibitor at a fixed NADPH (0.1 mM) concentrations. (x) $0.05 \mathrm{mM}$; ( $) 0.1 \mathrm{mM}$; (o) 0.15 $\mathrm{mM} ;\left(^{*}\right) 0.2 \mathrm{mM}$.

value of $0.372 \pm 0.06 \mathrm{mM}$. Kinetic studies on the inhibition of bovine kidney cortex GR by compound [2] (0.1; $0.2 ; 0.25 ; 0.3 \mathrm{mM}$ ) were performed. Compound [2] acted as a noncompetitive inhibitor with respect to GSSG (Fig. 3) and NADPH (Fig. 4). $K_{i}$ was calculated as $K_{i \mathrm{GSSG}}$ $0.431 \pm 0.03 \mathrm{mM}$ and $K_{\mathrm{NADPH}} 0.393 \pm 0.03 \mathrm{mM}$.

\section{Inhibition of bovine kidney cortex GR by hypolaetin 7-O-[6"'-O-acetyl- $\beta$-D-allopyranosyl- $(1 \rightarrow 2)]-\beta$-D- glucopyranoside [4]}

Compound [4] inhibited purified bovine kidney cortex GR, with an $\mathrm{IC}_{50}$ value of $0.297 \pm 0.05 \mathrm{mM}$. Kinetic characterization of the inhibition effects of the compound [4] $(0.1 ; 0.15 ; 0.2 ; 0.25 \mathrm{mM})$ on bovine kidney cortex GR was performed. This compound acted as a noncompetitive inhibitor with respect to GSSG (Fig. 5) and uncompetitive inhibitor with respect to NADPH (Fig. 6), and $K_{i}$ was calculated as $K_{i \mathrm{GSSG}} 0.360 \pm 0.02 \mathrm{mM}$ and $K_{\imath \mathrm{NADPH}} 0.167 \pm 0.02 \mathrm{mM}$, respectively.

Inhibition of bovine kidney cortex GR by isoscutellarein 7-O-[6"'-O-acetyl- $\beta$-D-allopyranosyl- $(1 \rightarrow 2)]-\beta$-Dglucopyranoside [5]

Compound [5] inhibited purified bovine kidney cortex $\mathrm{GR}$ with an $\mathrm{IC}_{50}$ value of $0.368 \pm 0.05 \mathrm{mM}$. The effects of compound [5] $(0.05 ; 0.1 ; 0.2 ; 0.3 \mathrm{mM})$ on GR from bovine kidney cortex were investigated using Lineweaver-Burk double reciprocal plot and a nonlinear curve fit- 


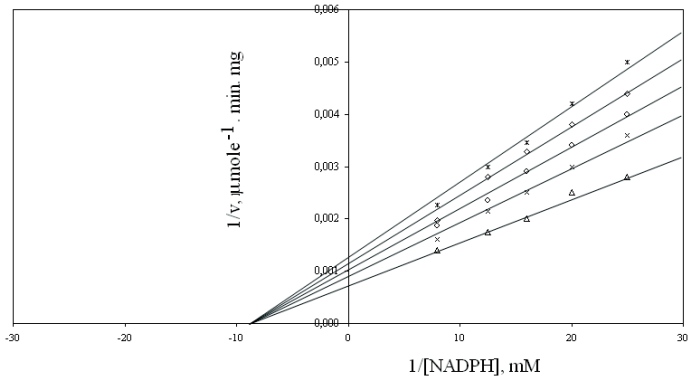

Figure 10. Inhibition of bovine kidney cortex glutathione reductase by 3'-hydroxy-4'-O-methylisoscutellarein 7-O-[6"'-Oacetyl- $\beta$-D-allopyranosyl- $(1 \rightarrow 2)]-\beta$-D-glucopyranoside.

Lineweaver-Burk double reciprocal plot of initial velocity against GSSG as varied substrate and compound [6] as inhibitor at a fixed GSSG (0.7 mM) concentrations. $(x) 0.05 \mathrm{mM} ;(\diamond) 0.1 \mathrm{mM} ;(0) 0.15$ $\mathrm{mM} ;\left(^{*}\right) 0.2 \mathrm{mM}$.

ting program. Compound [5] acted as an uncompetitive inhibitor with respect to GSSG (Fig. 7) and a noncompetitive inhibitor with respect to NADPH (Fig. 8). Inhibition constants were calculated as $K_{\mathrm{iGSSG}} 0.200 \pm 0.01$ $\mathrm{mM}$ and $K_{\mathrm{iNADPH}} 0.490 \pm 0.02 \mathrm{mM}$.

\section{Inhibition of bovine kidney cortex GR by $3^{\prime}$-hydroxy- 4'-O-methylisoscutellarein 7-O-[6"'-O-acetyl- $\beta$-D- allopyranosyl-( $1 \longrightarrow 2)]-\beta$-D-glucopyranoside [6]}

Compound [6] inhibited purified bovine kidney cortex GR with an $\mathrm{IC}_{50}$ value of $0.295 \pm 0.04 \mathrm{mM}$. Compound [6] $(0.05 ; 0.1 ; 0.15 ; 0.2 \mathrm{mM})$ acted as an uncompetitive inhibitor with respect to GSSG (Fig. 9) and a noncompetitive inhibitor with respect to NADPH (Fig. 10). $K_{i \mathrm{GSSG}}$ and $K_{\mathrm{NADPH}}$ were calculated as $0.169 \pm 0.01 \mathrm{mM}$ and $0.290 \pm 0.01 \mathrm{mM}$, respectively.

\section{DISCUSSION}

Natural products and their derivatives have historically been useful as a source of therapeutic agents (Koehn \& Carter, 2005). Natural product structures have long been recognized to possess characteristics of high chemical diversity, biochemical specificity and molecular diversity, which make them attractive targets for the discovery of new drugs (Ajay et al., 1998; Cao \& Kingston, 2009). In this study, natural products of Sideritis brevibracteata inhibited bovine kidney cortex GR. This enzyme is a significant target for the development of antimalarial and anticancer agents. Therefore, inhibition of this enzyme is an important research topic (Seefeldt et al., 2005). There is an urgent need for new, more affordable and accessible antimalarial agents. Natural products have played a dominant role in the discovery of drugs to treat human diseases (Batista et al., 2009).

In cancer therapy, inhibition of GR may be one of the important targets because this enzyme is key in the metabolism of glutathione, and its expression is often aberrant in human malignancies (Sun, 1990). Inhibition of this enzyme decreases GSH concentration in the cancer cell. Conjugation with glutathione of electrophilic xenobiotics facilitates their export from the cell and is a common detoxification mechanism, which may result in drug resistance. Therefore, a decreased GSH concentration will allow an increased effective drug concentration in the cell thus enhancing its cytotoxic effect on the cancer cell (Awasthi et al., 2009).
The phenolic compounds of $S$. brevibracteata inhibited bovine kidney cortex GR in a concentration-dependent manner. Compounds [1], [5] and [6] demonstrated similar inhibition kinetics, with an uncompetitive inhibition mode when GSSG was the varied substrate and a noncompetitive mode when NADPH was the varied substrate. However, compounds [2] and [4] showed different inhibition patterns. This can probably be attributed to these compounds' binding different amino acid residues or groups in the catalytic site of the enzyme. As is well known, uncompetitive inhibitors are thought to bind the enzyme-substrate complex and not the enzyme, but noncompetitive inhibition occurs when the inhibitor binds at a site away from the substrate-binding site, causing a reduction in the catalytic rate (Segel, 1975).

In conclusion, GR is a key enzyme that controls the cellular thiol-disulfide redox state in the cells, and it is essential for cellular homeostasis. During oxidative stress, intracellular GSSG accumulates, and the loss of thiol redox balance may cause deleterious consequences with regards to metabolic regulation, cellular integrity and organ homeostasis. Inhibition of GR and a disturbance in the cellular prooxidant-antioxidant balance may contribute to the genesis of many diseases. Its inhibitors have been shown to have anticancer and antimalarial activity by contributing to the reversal of drug resistance.

\section{Acknowledgement}

This work is a part of a project (0701101011) supported by the Hacettepe University Scientific Research Unit.

\section{REFERENCES}

Acan NL, Tezcan EF (1989) Sheep brain glutathione reductase: Purification and general properties. FEBS Lett 250: 72-74.

Ajay A, Walters WP, Murcko MA (1998) Can we learn to distinguish between drug like and nondrug-likemolecules? I Med Chem 41: 3314-3324.

Arscott LD, Veine DM, Williams CH Jr. (2000) Mixed disulfide with glutathione as an intermediate in the reaction catalyzed by glutathione reductase from yeast and as a major form of the enzyme in the cell. Biochemistry 39: 4711-4721.

Awasthi YC, Chaudhary P, Vatsyayan R, Sharma A, Awasthi S, Sharma R. (2009) Physiological and pharmacological significance of glutathione-conjugate transport. I Toxicol Environ Health B Crit Rev 12: $540-551$.

Barberan FA. Manez S, Villar A (1987) Identification of antiinflammatory agents from Sideritis species growing in Spain. J Nat Prod 50: 313-314.

Baser KHC, Kirimer N, Tümen G (1997) The Essential Oil of Sideritis scardica Griseb. subsp. scardica. J Essent Oil Res 9: 205-207.

Batista R, Silva Ade J Jr, de Oliveira AB (2009) Plant-derived antimalarial agents: new leads and efficient phytomedicines. Part II. Nonalkaloidal natural products. Molecules 14: 3037-3072.

Bauer H, Fritz-Wolf K, Winzer A, Kuhner S, Little S, Yardley V, Vezin H, Palfey B, Schirmer RH, Davioud-Charvet E. (2006) A fluoro analogue of the menadione derivative 6-[2'-(3'-methyl)-1',4'naphthoquinolyl] hexanoic acid is a suicide substrate of glutathione reductase. Crystal structure of the alkylated human enzyme. $J A m$ Chem Soc 128: 10784-10794.

Cao S, Kingston DG (2009) Biodiversity conservation and drug discovery: Can they be combined? The Suriname and Madagascar experiences. Pharm Biol 47: 809-823.

De Las Heras B, Vivas JM, Villar A (1994) Anti-inflammatory activity of Sideritis javalambrensis extracts. J Ethnopharmacol 41: 15-17.

Ezer N, Sakar M K, Rodriguez B, Dela Torre MC (1992) Flavonoid glycosides and a phenylpropanoid glycoside from Sideritis perfoliata. Int J Pharmacog 30: 61-65.

Güvenç A, Okada Y, Küpeli Akkol E, Duman H, Okuyama T, Çalıș I (2010) Investigations of anti-inflammatory, antinociceptive, antioxidant and aldose reductase inhibitory activities of phenolic compounds from Sideritis brevibracteata. Food Chem 118: 686-692.

Koehn FE, Carter GT. (2005) The evolving role of natural products in drug discovery Nature Reviews Drug Discovery 4: 206-220. 
Krauth-Siegel RL, Arscott LD, Schonleben-Janas A, Schirmer RH, Williams CHJr. (1998) Role of active site tyrosine residues in catalysis by human glutathione reductase. Biochemistry 37: 13968-13977.

Lee KH (2010) Discovery and development of natural product-derived chemotherapeutic agents based on a medicinal chemistry approach. J Nat Prod 73: 500-516.

Seefeldt T, Dwivedi C, Peitz G, Herman J, Carlson L, Zhang Z, Guan XJ (2005) Acetylamino-3-[4-(2-acetylamino-2-carboxyethylsulfanylcarbonylamino)-phenylcarbamoylsulfanyl] propionic acid and its derivatives as a novel class of glutathione reductase inhibitors. Med Chem 48: 5224-5231.

Segel IH (1975) Enzyme Kinetics. 3rd edn, Chapter 3. pp 100-159. John Wiley and Sons: Toronto.
Sun Y (1990) Free radicals, antioxidant enzymes, and carcinogenesis. Free Radic Biol Med 8: 583-599.

Tandogan B, Ulusu NN (2010) Purification and kinetics of bovine kidney cortex glutathione reductase. Protein Pept Lett 17: 667-674.

Untucht-Grau R, Schirmer RH, Schirmer I, Krauth-Siegel RL (1981) Glutathione reductase from human erythrocytes: amino-acid sequence of the structurally known FAD-binding domain. Eur J Biochem 120: 407-419.

Yesilada E, Ezer N (1989) The antiinflammatory activities of some Sideritis spp. growing in Turkey. Int J Crude Drug Res 27: 38-40. 